\title{
ChemComm
}

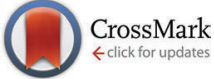

Cite this: Chem. Commun., 2017. 53,2339

Received 5th December 2016, Accepted 27th January 2017

DOI: $10.1039 / \mathrm{c} 6 \mathrm{cc} 09510 \mathrm{k}$

rsc.li/chemcomm

\section{Firmly anchored photosensitizer Chlorin e6 to layered double hydroxide nanoflakes for highly efficient photodynamic therapy in vivo $\dagger$}

\author{
Li Yan, ${ }^{\text {ab }}$ Zhigang Wang, ${ }^{c}$ Xianfeng Chen, ${ }^{\star d}$ Xiao-Jun Gou, ${ }^{a}$ Zhenyu Zhang, ${ }^{b}$ \\ Xiaoyue Zhu, ${ }^{b}$ Minhuan Lan, ${ }^{\mathrm{b}}$ Wei Chen, ${ }^{\mathrm{b}}$ Guangyu Zhu ${ }^{\mathrm{c}}$ and Wenjun Zhang ${ }^{\star \mathrm{b}}$
}

\begin{abstract}
In using nanomaterials for advanced photodynamic therapy, it is important to improve surface modification to increase stability, minimize non-specific loss of drugs in blood circulation, enhance the photostability of photosensitizers, and achieve highly efficient intracellular uptake. In this report, we for the first time covalently conjugate photosensitizer Chlorin e6 (Ce6) to polyethylene glycol modified layered double hydroxides and produce hybrid nanoflakes. These nanoflakes display many superior characteristics and lead to excellent in vivo photodynamic therapeutic efficiency and safety profiles.
\end{abstract}

Photo-induced therapy, like photodynamic therapy (PDT), is a promising approach for cancer treatment, because of its high efficiency, low toxicity and minimal invasion. ${ }^{1-9}$ In a typical PDT treatment, photosensitizers (PSs) play a key role by generating singlet oxygen $\left({ }^{1} \mathrm{O}_{2}\right)$ to induce the apoptosis/necrosis of local cancer cells. ${ }^{10,11}$ Chlorin e6 (Ce6) is a widely used PS molecule. ${ }^{12,13}$ Similar to many other conventional PS molecules, Ce6 suffers from major limitations such as low solubility, intracellular delivery efficiency, and photostability. To address the problems of free drug molecules, nanomaterials have been developed and have attracted great attention in cancer therapy. ${ }^{14-20}$ For example, polymer nanoparticles, upconversion nanoparticles, gold nanostructures, and silica nanoparticles have been employed in delivering PS molecules. ${ }^{21-24}$ Among the choices, layered double hydroxide (LDH) nanomaterials have been very attractive due to their

\footnotetext{
${ }^{a}$ Antibiotics Research and Re-evaluation Key Laboratory of Sichuan Province, Sichuan Industrial Institute of Antibiotics (SIIA), Chengdu University, Chengdu, Sichuan, P. R. China

${ }^{b}$ Center of Super-Diamond and Advanced Films (COSDAF) and Department of Physics and Materials Science, City University of Hong Kong, Hong Kong SAR, P. R. China. E-mail: apwjzh@cityu.edu.hk

${ }^{c}$ Department of Biology and Chemistry, City University of Hong Kong, Hong Kong SAR, P. R. China

${ }^{d}$ Institute for Bioengineering, School of Engineering, The University of Edinburgh, King's Buildings, Mayfield Road, Edinburgh EH9 3JL, UK.

E-mail: xianfeng.chen@oxon.org

$\dagger$ Electronic supplementary information (ESI) available: See DOI: 10.1039/c6cc09510k
}

superior merits including good biocompatibility, biodegradability like biomolecule based nano-carriers, ${ }^{25-28}$ and $\mathrm{pH}$ sensitive drug release characteristics. ${ }^{29-44}$ Despite these advantages, there are also issues when LDH nanoparticles are employed in delivering PS molecules for photodynamic therapy. In particular, owing to the lack of functional groups on the surface, these nanomaterials are not stable in blood circulation. Additionally, drug molecules are commonly loaded into the interlayers through an ionexchange method. In such a system, drug molecules can very easily and quickly release out once in blood circulation, thereby causing undesirable side effects and reduced therapeutic efficacy.

To overcome these drawbacks, in this report, we create new LDH-Ce6 hybrid nanoflakes by functionalizing the surface of LDH nanoparticles and covalently conjugate Ce6 molecules. Using this strategy, Ce6 molecules are firmly anchored to the stabilized LDH matrix. In this system, Ce6 molecules tightly bind to LDH with very slow release profiles, avoiding premature release of the drug molecules in blood and ensuring their efficient delivery to tumors. Once there, the acidic environment in the tumors will significantly expedite the drug release for powerful PDT.

The schematic of the preparation of LDH-Ce6 hybrid nanoflakes is illustrated in Scheme S1 (ESI $\dagger$ ). The interlayer spacing of LDH nanoparticles was first expanded by addition of sodium dodecyl sulfate (SDS) during preparation. ${ }^{45}$ Through this approach, the interlayer spacing of LDH nanoparticles increases from 0.77 to $2.6 \mathrm{~nm}$, indicated by the (003) diffraction peak's shift from $11.5^{\circ}$ to $3.4^{\circ}$ in the X-ray diffraction (XRD) pattern (Fig. S1a, ESI $\dagger$ ). In Fourier transform infrared (FTIR) spectra, the appearance of SDS' peaks of SDS expanded LDH nanoparticles confirms the successful incorporation of SDS into LDH (Fig. S1b and S2, ESI $\dagger$ ). Next, Ce6 molecules were covalently linked to (3-aminopropyl)triethoxysilane (APTES) in the presence of $N$-(3-dimethylaminopropyl)- $N$-ethylcarbodiimide hydrochloride (EDC) as a catalyst (Scheme S1, ESI $\dagger$ ). Finally, to get LDH-Ce6 hybrid nanoflakes, APTES linked Ce6 was added to methylene chloride solution containing SDS expanded LDH nanoparticles and $N$-cetyl- $N, N, N$-trimethylammonium (CTAB) 


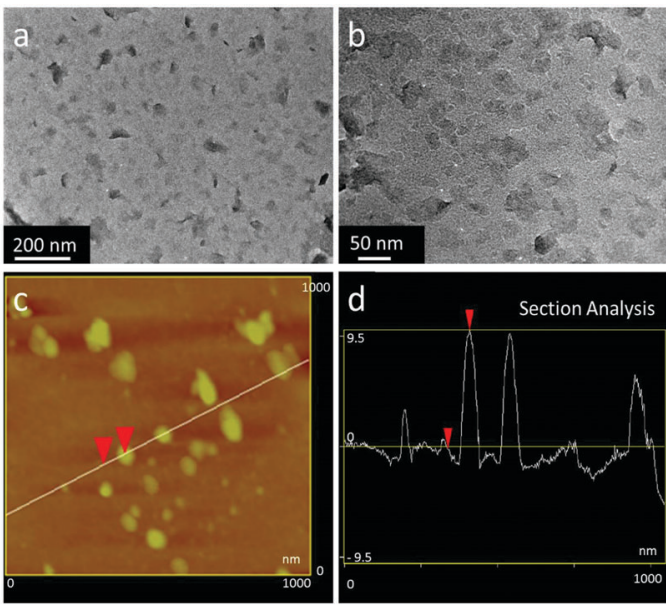

Fig. 1 ( $a$ and b) TEM images of LDH-Ce6 hybrid nanoflakes. (c) AFM image of $\mathrm{LDH}-\mathrm{Ce} 6$ hybrid nanoflakes. (d) The thickness analysis of $\mathrm{LDH}-\mathrm{Ce} 6$ hybrid nanoflakes along the marked line in (c).

followed by centrifugation. As shown in Fig. S2 (ESI $\dagger$ ), the as-prepared LDH-Ce6 hybrid nanoflakes contain FTIR peaks of both $\mathrm{LDH}$ and Ce6, demonstrating the successful attachment of Ce6 to the LDH matrix. Moreover, the two broad peaks at around $1000-1200 \mathrm{~cm}^{-1}$ correspond to $\mathrm{Si}-\mathrm{O}-\mathrm{Si}$ vibration, indicating the covalent attachment of APTES to the LDH matrix (Fig. S1b, ESI $\dagger$ ). ${ }^{46}$ Fig. 1a and b shows the transmission electron microscopy (TEM) images of LDH-Ce6 hybrid nanoflakes with a size of about 20-50 nm. These nanostructures were further characterized by atomic force microscopy (AFM) (Fig. 1c and d). The morphology of LDH-Ce6 hybrid nanoflakes is very similar to what we obtained by TEM. The thickness is about $10 \mathrm{~nm}$, indicating that the pristine LDH structure was partially exfoliated to hybrid nanoflakes. The diminished diffraction peaks in the LDH-Ce6 XRD pattern (Fig. S1a, ESI $\dagger$ ) also confirm the destruction of the original layered structure of the pristine LDH nanoparticles.

After successfully fabricating LDH-Ce6 hybrid nanoflakes, we studied their optical properties and ability of singlet oxygen production. Fig. S3 (ESI $\dagger$ ) shows the absorbance spectra of free Ce6 and LDH-Ce6 hybrid nanoflakes. After Ce6 molecules are incorporated into $\mathrm{LDH}$, a slight red-shift of its absorption peaks can be observed. Other than the trivial shift, the absorption peaks are largely maintained, indicating that the whole fabrication process does not destruct the optical properties of Ce6 molecules. To demonstrate that the LDH-Ce6 hybrid nanoflakes have the potential for photodynamic therapy, we used 1,3-diphenylisobenzofuran (DPBF) as a chemical probe to detect generated singlet oxygen $\left({ }^{1} \mathrm{O}_{2}\right)$. As shown in Fig. S4a (ESI $\left.\dagger\right)$, both $\mathrm{Ce} 6$ and $\mathrm{LDH}-\mathrm{Ce} 6$ hybrid nanoflakes can effectively generate ${ }^{1} \mathrm{O}_{2}$ at $\mathrm{pH} 7.4$ and $\mathrm{pH}$ 5.0, which is indicated by the dramatically decreased absorption intensity of DPBF. It is clear that the hybrid nanoflakes maintain their ability of singlet oxygen generation. Particularly, at $\mathrm{pH}$ 5.0, the production of singlet oxygen is more or less similar to that from free Ce6 molecules. In great contrast, the conventional method of loading Ce6 into the interlayers of $\mathrm{LDH}$ nanoparticles by ion-exchange causes dramatically decreased ${ }^{1} \mathrm{O}_{2}$ generation efficiency. $^{31}$
Furthermore, we studied the photostability of $\mathrm{Ce} 6$ and LDH-Ce6 hybrid nanoflakes by exposing both materials to $650 \mathrm{~nm}$ light illumination for different periods of time (Fig. S5, $\mathrm{ESI} \dagger$ ). When Ce6 solution is exposed to the light, the absorbance intensity rapidly falls. This suggests that serious photobleaching has occurred to the PS molecules. In comparison, the decreasing rate of the absorbance of the hybrid nanoflakes is much slower. From these observations, it is apparent that Ce6 can be protected by LDH hybrid nanoflakes and therefore the photostability is improved. To further demonstrate the enhanced photostability of LDH-Ce6 hybrid nanoflakes, we also studied the ${ }^{1} \mathrm{O}_{2}$ generation ability of free Ce6 and LDH-Ce6 hybrid nanoflakes after exposing both materials to $650 \mathrm{~nm}$ light irradiation for $30 \mathrm{~min}$ before the test (Fig. S4b, ESI $\dagger$ ). Very attractively, LDH-Ce6 can still effectively yield ${ }^{1} \mathrm{O}_{2}$. In sharp contrast, free Ce6 molecules nearly lose their ability to produce ${ }^{1} \mathrm{O}_{2}$. This test provides strong evidence that LDH-Ce6 hybrid nanoflakes are much more stable than free Ce6 under light irradiation, which makes LDH-Ce6 hybrid nanoflakes very promising for photodynamic therapy.

After having explored that LDH-Ce6 hybrid nanoflakes preserve their ability to yield singlet oxygen and exhibit much higher photostability than free Ce6, we then investigated whether Ce6 is firmly anchored to the LDH matrix in biological environments. In this experiment, $\mathrm{LDH}-\mathrm{Ce} 6$ hybrid nanoflakes were dispersed in $\mathrm{pH} 7.4$ phosphate buffered saline (PBS). During the observation period of 72 hours, only a small fraction of Ce6 is released, implying that most Ce6 molecules robustly bind to LDH nanostructures at pH 7.4 (Fig. S6, ESI $\dagger$ ). Thus, we prove that $\mathrm{Ce} 6$ molecules strongly bind to $\mathrm{LDH}$ nanostructures and remain stable at $\mathrm{pH} 7.4$ with minimal liberation. In sharp contrast, when drug molecules are loaded into $\mathrm{LDH}$ nanoparticles using an ion-exchange method, more than $60 \%$ can be released during the first 24 hours. ${ }^{47}$ This leads to serious non-specific loss of $\mathrm{Ce} 6$ in blood transport, resulting in low therapeutic efficiency and high toxicity.

Besides that LDH can increase the photostability and stability of Ce6 in biological environments, enhanced cellular uptake would be another advantage of the LDH based system. Fig. S7 (ESI $\dagger$ ) presents the confocal microscopy images of A549 cells incubated with free Ce6 and LDH-Ce6 hybrid nanoflakes for 4 hours and 24 hours. From these pictures, very low fluorescence signals can be detected from A549 cells incubated with free Ce6 for 4 and 24 hours, showing that not many free Ce6 molecules penetrate the cell membrane and are delivered into cells. In comparison, strong fluorescence signals are observed for the cells treated with LDH-Ce6 hybrid nanoflakes for the same duration. These results unambiguously imply that the LDH-Ce6 hybrid nanoflakes can efficiently transport Ce6 molecules to cells. We subsequently analyzed the ability of $\mathrm{LDH}-\mathrm{Ce} 6$ hybrid nanoflakes to generate ${ }^{1} \mathrm{O}_{2}$ within cells by measuring cellular reactive oxygen species (ROS). In this experiment, A549 cells were incubated with $\mathrm{Ce} 6$ and $\mathrm{LDH}-\mathrm{Ce} 6$ hybrid nanoflakes for 4 and 24 hours followed by confocal microscopy observation (Fig. 2 and Fig. S8-S10, ESI $\dagger$ ). It is very obvious that only a very small amount of ROS can be detected from A549 cells incubated with free Ce 6 for both 4 and 24 hours, because a trivial 


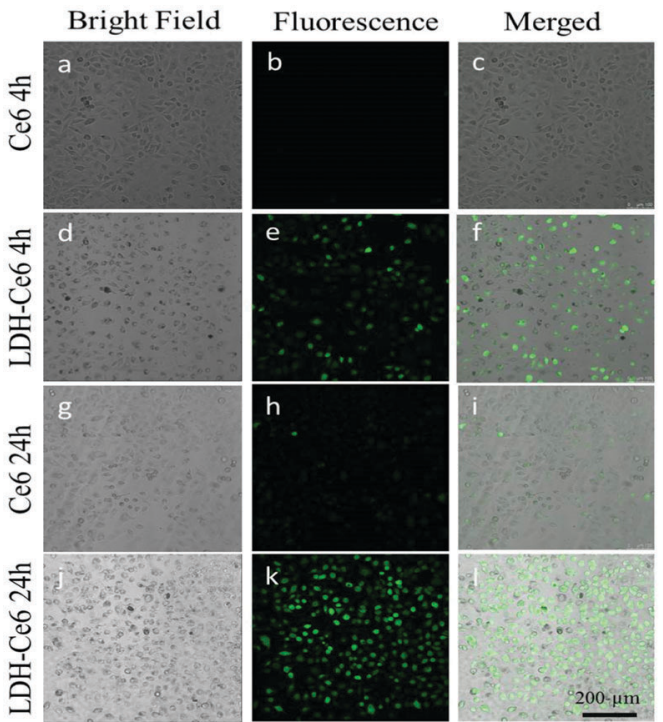

Fig. 2 Laser scanning confocal microscopy images of H2DCFDA-stained A549 cells after $4(a-f)$ and $24(g-l)$ hours of incubation with Ce6 and $\mathrm{LDH}-\mathrm{Ce} 6$ hybrid nanoflakes, and followed by 10 minutes of $650 \mathrm{~nm}$ irradiation.

amount of Ce6 is delivered into cells when free Ce6 molecules are applied. On the contrary, in the two groups of A549 cells mixed with LDH-Ce6 hybrid nanoflakes, the ROS production is very high, marked by bright fluorescence. This significantly elevated ROS production is likely due to the enhanced cellular internalization of $\mathrm{LDH}-\mathrm{Ce} 6$ hybrid nanoflakes.

The phototoxicity of Ce6 and LDH-Ce6 hybrid nanoflakes was then studied to assess their cancer cell killing efficacy (Fig. 3a and b and Fig. S11, ESI $\dagger$ ). Similar to the methodology in cellular uptake and ROS production analysis, A549 cells were incubated with Ce6 and LDH-Ce6 hybrid nanoflakes for 4 and 24 hours under light illumination. When A549 cells are incubated with free Ce6 for 4 hours, the cell viability is not negatively affected, even at the highest concentration of $5 \mu \mathrm{g} \mathrm{mL} \mathrm{m}^{-1}$. When the incubation time of $\mathrm{Ce} 6$ is extended to 24 hours, the viability of A549 cells decreases below $10 \%$ at a concentration of $5 \mu \mathrm{g} \mathrm{mL}$. This can be explained by the observation of cellular uptake and ROS detection: incubation of $4 \mathrm{~h}$ is not enough for free Ce6
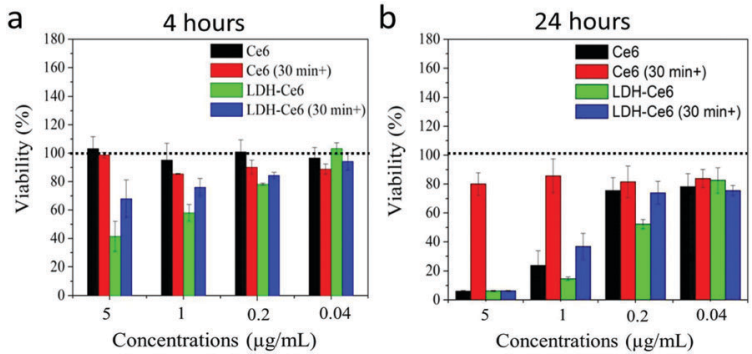

Fig. 3 The phototoxicity of free $\mathrm{Ce} 6$ (Ce6), $\mathrm{LDH}-\mathrm{Ce} 6$ hybrid nanoflakes (LDH-Ce6), 30 minutes $650 \mathrm{~nm}$ light pre-irradiated free Ce6 (Ce6 (30 $\mathrm{min}+))$ and 30 minutes $650 \mathrm{~nm}$ light pre-irradiated LDH-Ce6 hybrid nanoflakes (LDH-Ce6 (30 min+)). A549 cells were incubated for 4 hours (a) and 24 hours (b), followed by 10 minutes of light irradiation. molecules to be delivered into cytoplasm, and therefore negligible cellular ROS was generated to kill the cells. In comparison, the viability of A549 cells treated with LDH-Ce6 hybrid nanoflakes containing the same concentration of Ce6 for 4 hours sharply falls below $50 \%$. This means that, with the aid of LDH, Ce6 starts to show obvious function at a much earlier time.

We also studied the photostability of our fabricated LDH-Ce6 hybrid nanoflakes with free Ce6 as a reference by measuring their cell killing efficacy. In this study, free Ce6 and LDH-Ce6 hybrid nanoflakes were irradiated under $650 \mathrm{~nm}$ light for 30 minutes before incubation with A549 cells. After the pre-irradiation, the materials were incubated with cells for 4 and $24 \mathrm{~h}$ followed by MTT assay. Within our expectation, free Ce6 did not show any phototoxicity to A549 cells, even in the case of $5 \mu \mathrm{g} \mathrm{mL}{ }^{-1}$ concentration and $24 \mathrm{~h}$ incubation. These data are consistent with our previous discovery that Ce6 is not stable upon light exposure and the ability of ${ }^{1} \mathrm{O}_{2}$ can be almost lost with only 30 minutes of light irradiation. Attractively, LDH-Ce6 hybrid nanoflakes can still maintain their ability to kill cancer cells. As shown in Fig. 3b, the cell viabilities still display dependency on the dose of LDH-Ce6. When the concentration of the hybrid nanoflakes increases from 0.04 to $5 \mu \mathrm{g} \mathrm{mL}{ }^{-1}$, nearly all cells are killed. Collectively, these results show that LDH-Ce6 hybrid nanoflakes exhibit excellent cancer cell killing efficiency and also confirm their high photostability.

To demonstrate the therapeutic efficacy of our LDH-Ce6 hybrid nanoflakes in vivo, their antitumor activity was assessed on $4 \mathrm{~T} 1$ tumor bearing BALB/C mice. In order to prolong in vivo circulation time and reduce non-specific loss of LDH-Ce6, we co-modified the surface of our LDH nanostructures with PEG-carboxyl molecules $\left(M_{\mathrm{w}}=5000\right)$ simultaneously with Ce6 (Fig. S12, ESI $\dagger$ ). The mice were intravenously injected with PBS, the LDH-Ce6 mix (LDH loaded into the interlayers of LDH through ion-exchange), free $\mathrm{Ce} 6, \mathrm{LDH}-\mathrm{Ce} 6$ hybrid nanoflakes on days 1, 4 and 7, and exposed to $650 \mathrm{~nm}$ light on days 1, 3, 5, 7, 9 and 11. The results are summarized in Fig. 4a. Attractively, it can be seen that LDH-Ce6 hybrid nanoflakes achieve significantly improved tumor growth inhibition as indicated by the size reduction to $13.4 \%, 14.3 \%$ and $26.9 \%$ of the tumor volume in the PBS, LDH-Ce6 Mix, free Ce6 treated groups, respectively. Moreover, we found that the LDH-Ce6 mix does not seem to have therapeutic efficacy with intravenous injection. This sharp
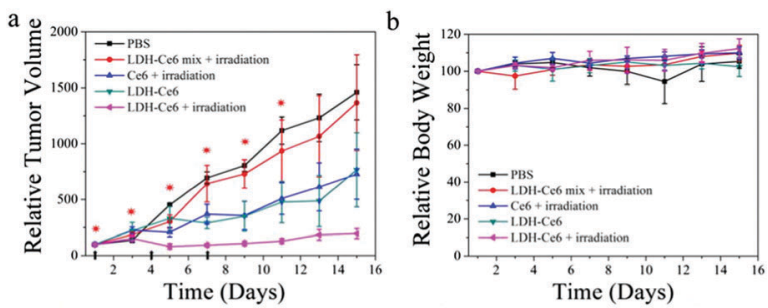

Fig. 4 (a) Tumor growth curves and (b) the body weight evolution of 4T1 tumor bearing BALB/C mice after intravenous injection with different formulations on days 1,4 , and 7 and sacrifice on day 15 (the arrows on the $X$-axis indicate tail vein injection on days 1,4 and $7 ;$ * indicates light irradiation on days $1,3,5,7,9$ and 11). 
difference demonstrates that our LDH-Ce6 hybrid nanoflakes can overcome the major disadvantages of the traditional LDH-PS system which loads PS simply by ion-exchange. LDH-Ce6 without irradiation seems to also have some therapeutic efficacy. The reason might be that all mice are in an IVC (Individually Ventilated Cages) animal house with white room light and it may help to generate a small amount of ROS (Fig. S13, ESI $\dagger$ ). In addition, the mice treated with LDH-Ce6 hybrid nanoflakes steadily gain body weight, which partially indicates that the hybrid nanoflakes have minimal side-effects. Moreover, no obvious organ damage was found by hematoxylin and eosin (H\&E) staining of major organs after the mice were treated with LDH-Ce6 hybrid nanoflakes (with $650 \mathrm{~nm}$ light irradiation), which further confirms the biosafety of LDH-Ce6 hybrid nanoflakes (Fig. S14, ESI $\dagger$ ).

In summary, we created an approach to covalently bond $\mathrm{Ce} 6$ molecules to LDH with PEG surface modification for highly efficient anticancer photodynamic therapy. The LDH-Ce6 hybrid nanoflakes have the ability to generate singlet oxygen, high photostability, efficient intracellular delivery, and ultimately superior in vitro and in vivo photodynamic therapeutic efficacy with minimal side-effects.

This work was financially supported by the General Research Fund of Hong Kong (GRF No. 11338516), the National Natural Science Foundation of China (No. 51672230 and 51372213), the School of Engineering of University of Edinburgh, and the Royal Society Research Grant Scheme RG150564.

\section{Notes and references}

1 X. Liang, X. Li, X. Yue and Z. Dai, Angew. Chem., Int. Ed., 2011, 50, 11622.

2 R. Liang, R. Tian, L. Ma, L. Zhang, Y. Hu, J. Wang, M. Wei, D. Yan, D. G. Evans and X. Duan, Adv. Funct. Mater., 2014, 24, 3144.

3 R. Chen, J. Zhang, Y. Wang, X. Chen, J. A. Zapien and C. S. Lee, Nanoscale, 2015, 7, 17299.

4 Y. Yang, X. Zhang, C. Yu, X. Hao, J. Jie, M. Zhou and X. Zhang, Adv. Healthcare Mater., 2014, 3, 906-915.

5 W. Li, X. Zhang, M. Zhou, B. Tian, C. Yu, J. Jie, X. Hao and X. Zhang, Adv. Healthcare Mater., 2014, 3, 1475.

6 L. Yan, J. Zhang, C. S. Lee and X. Chen, Small, 2014, 10, 4487.

7 J. Ge, M. Lan, B. Zhou, W. Liu, L. Guo, H. Wang, Q. Jia, G. Niu, X. Huang, H. Zhou, X. Meng, P. Wang, C. S. Lee, W. Zhang and X. Han, Nat. Commun., 2014, 5, 4596.

8 X. Zhuang, X. Ma, X. Xue, Q. Jiang, L. Song, L. Dai, C. Zhang, S. Jin, K. Yang, B. Ding, P. C. Wang and X. J. Liang, ACS Nano, 2016, 10, 3486

9 Y. Huang, S. He, W. Cao, K. Cai and X. J. Liang, Nanoscale, 2012, 4, 6135.

10 R. F. Ronnelly, P. A. McCarron, C. M. Cassidy, J. S. Elnorn and M. M. Tunney, J. Controlled Release, 2007, 117, 217.

11 J. Zhang, Y. C. Liang, X. Lin, X. Zhu, L. Yan, S. Li, X. Yang, G. Zhu, A. L. Rogach, P. K. Yu, P. Shi, L. C. Tu, C. C. Chang, X. Zhang, X. Chen, W. Zhang and C. S. Lee, ACS Nano, 2015, 9, 9741.

12 J. F. Zhang, F. F. An, Y. N. Li, C. J. Zheng, Y. L. Yang, X. J. Zhang and X. H. Zhang, Chem. Commun., 2013, 49, 8072.

13 Q. Chen, X. Wang, C. Wang, L. Feng, Y. Li and Z. Liu, ACS Nano, $2015,9,5223$.
14 M. J. Zhou, X. J. Zhang, Y. Y. Yang, Z. Liu, B. S. Tian, J. J. Jie and X. H. Zhang, Biomaterials, 2013, 34, 8960.

15 X. H. Diao, W. Li, J. Yu, X. J. Wang, X. J. Zhang, Y. L. Yang, F. F. An, Z. Liu and X. H. Zhang, Nanoscale, 2015, 4, 5373.

16 X. Du, X. Y. Li, L. Xiong, X. J. Zhang, F. Kleitz and S. Z. Qiao, Biomaterials, 2016, 91, 90.

17 L. Xiong, X. Du, F. Kleitz and S. Z. Qiao, Small, 2015, 11, 5919.

18 W. Li, X. Zhang, X. Hao, J. Jie, B. Tian and X. Zhang, Chem. Commun., 2013, 49, 10989.

19 A. Popat, B. P. Ross, J. Liu, S. Jambhrunkar, F. Kleitz and S. Z. Qiao, Angew. Chem., Int. Ed., 2012, 51, 12486.

20 A. P. Raphael, J. P. Sisney, D. C. Liu and T. W. Prow, Curr. Drug Delivery, 2015, 12, 78.

21 Q. Jia, J. Ge, W. Liu, S. Liu, G. Niu, L. Guo, H. Zhang and P. Wang, Nanoscale, 2016, 8, 13067.

22 G. Yang, H. Gong, X. Qian, P. Tan, Z. Li, T. Liu, J. Liu, Y. Li and Z. Liu, Nano Res., 2015, 8, 751.

23 Z. Li, C. Wang, L. Cheng, H. Gong, S. Yin, Q. Gong, Y. Li and Z. Liu, Biomaterials, 2013, 34, 9160.

24 X. Chen, D. Peng, Q. Ju and F. Wang, Chem. Soc. Rev., 2015, 44, 1318.

25 K. Liu, R. Xing, Q. Zou, G. Ma, H. Möhwald and X. Yan, Angew. Chem., Int. Ed., 2016, 55, 3036.

26 N. Zhang, F. Zhao, Q. Zou, Y. Li and X. Yan, Small, 2016, 12, 5936.

27 D. Hu, Z. Sheng, G. Gao, G. Siu, C. Liu, Q. Wan, P. Gong, H. Zheng, Y. Ma and L. Cai, Biomaterials, 2016, 93, 10.

28 R. Xing, K. Liu, T. Jiao, N. Zhang, K. Ma, R. Zhang, Q. Zou, G. Ma and X. Yan, Adv. Mater., 2016, 28, 3669.

29 Z. Gu, J. J. Atherton and Z. P. Xu, Chem. Commun., 2015, 51, 3024.

30 L. Li, W. Gu, J. Liu, S. Yan and Z. Xu, Nano Res., 2015, 8, 682.

31 Z. Wang, R. Ma, L. Yan, X. Chen and G. Zhu, Chem. Commun., 2015, 51, 11587.

32 W. Chen, B. Zhang, T. Mahony, W. Gu, B. Rolfe and Z. P. Xu, Small, 2016, 12, 1627.

33 H. Zuo, Z. Gu, H. Cooper and Z. P. Xu, J. Colloid Interface Sci., 2015, 459, 10.

34 J. Z. Zhou, Y. Liang, J. Zhang, L. Li, Y. Xu, X. Ruan, G. Qian and Z. P. Xu, J. Hazard. Mater., 2014, 279, 141.

35 J. Liu, R. Harrison, J. Z. Zhou, T. T. Liu, C. Yu, G. Q. Lu, S. Z. Qiao and Z. P. Xu, J. Mater. Chem., 2011, 21, 10641.

36 L. Yan, A. P. Raphael, X. Zhu, B. Wang, W. Chen, T. Tang, Y. Deng, H. J. Sant, G. Zhu, K. W. Choy, B. K. Gale, T. W. Prow and X. Chen, Adv. Healthcare Mater., 2014, 3, 555.

37 L. Yan, Y. Yang, W. Zhang and X. Chen, Adv. Mater., 2014, 26, 5533.

38 L. Yan, W. Chen, X. Zhu, L. Huang, Z. Wang, G. Zhu, V. A. L. Roy, K. N. Yu and X. Chen, Chem. Commun., 2013, 49, 10938.

39 S. Yan, B. E. Rolfe, B. Zhang, Y. H. Mohammed, W. Gu and Z. P. Xu, Biomaterials, 2014, 35, 9508.

40 Y. Kuo, Y. Kuthati, R. K. Kankala, P. Wei, C. Weng, C. Liu, P. Sung, C. Mou and C. Lee, J. Mater. Chem. B, 2015, 3, 3447.

41 Z. Gu, B. E. Rolfe, Z. P. Xu, J. H. Campbell, G. Q. Lu and A. C. Thomas, Adv. Healthcare Mater., 2012, 1, 669.

42 L. Li, W. Gu, J. Chen, W. Chen and Z. P. Xu, Biomaterials, 2014, 35, 3331.

43 Z. Gu, H. Zuo, A. Wu and Z. P. Xu, J. Controlled Release, 2015, 213, e150.

44 Z. P. Xu, G. S. Stevenson, C. Q. Lu, G. Q. Lu, P. F. Bartlett and P. P. Gray, J. Am. Chem. Soc., 2006, 128, 36.

45 L. Yan, Y. Wang, J. Li, S. Kalytchuk, A. S. Susha, S. V. Kershaw, F. Yan, A. L. Rogach and X. Chen, J. Mater. Chem. C, 2014, 2, 4490.

46 Y. Wang, Y. Wang, P. Gao, Y. Li and H. Li, CrystEngComm, 2011, 13, 177.

47 R. Ma, Z. Wang, L. Yan, X. Chen and G. Zhu, J. Mater. Chem. B, 2014, 2,4868 . 